\title{
Determinación de resistencia antihelmíntica frente a ivermectina de nematodos del bovino en dos predios del sur de Chile
}

\author{
Anthelminthic resistance of bovine nematodes against ivermectin \\ in two farms of the south of Chile \\ G Sievers $^{1 *}$, A Alocilla ${ }^{1}$ \\ ${ }^{1}$ Instituto de Patología Animal, Universidad Austral de Chile, Valdivia, Chile.
}

\begin{abstract}
SUMMARY
Anthelminthic resistance of bovine nematodes against ivermectin was determined in two farms in the south of Chile: one of them located near Purranque (Farm 1) and the other near San Pablo (Farm 2), in which 14 and 22 bovines aged 7 to 8 months were treated with $0.2 \mathrm{ml} / \mathrm{kg}$ of ivermectin, respectively. Faecal samples were obtained from each animal 7 days before and 21 days after the treatment to carry out the faecal egg count reduction test (FECRT). A 90.3\% of egg count reduction was found in Farm 1, with Trichostrongylus and Cooperia as the most resistant genera. In Farm 2 there was $73.5 \%$ of egg count reduction, with Nematodirus as the most resistant genera followed by Cooperia and Trichostrongylus. It was concluded that there was anthelminthic nematode resistance to ivermectin in Farm 2, while in Farm 1 resistance was gradually appearing.
\end{abstract}

Palabras clave: resistencia antihelmíntica, ivermectina, bovinos.

Key words: anthelminthic resistance, ivermectin, bovine.

\section{INTRODUCCION}

El control eficiente de las parasitosis de los bovinos se puede lograr con un manejo adecuado de las superficies de pastoreo y el uso estratégico y mínimo de antiparasitarios. Sin embargo, en la práctica productiva se ha instaurado la administración regular de antiparasitarios como una rutina que se realiza incontroladamente y sin ningún criterio técnico. Este hecho es la principal causa de un aumento de la resistencia antihelmíntica de los parásitos.

Se considera que hay resistencia cuando la efectividad de un fármaco cesa o disminuye. Ello se produce porque después de cada tratamiento sobrevive un pequeño número de individuos que son resistentes al fármaco utilizado, y son los únicos que logran reproducirse y contaminar las pasturas con sus huevos (Jackson 1993). Con la continua selección de los individuos resistentes que se produce por el uso repetido de los antiparasitarios, aumenta la frecuencia de los genes de la resistencia en una población, hasta producir el reemplazo de la población sensible por una población resistente al fármaco con el consiguiente fracaso del tratamiento antihelmíntico (Romero y col 1998, Sangster 1999). El establecimiento de una población resistente a

Aceptado: 06.06.2006.

* E-mail: gsievers@uach.cl; Casilla 567, Valdivia, Chile. un antihelmíntico es un proceso de carácter irreversible.

La resistencia antihelmíntica frente a los bencimidazoles, probencimidazoles, levamisol, morantel y avermectinas se presenta especialmente en los equinos, ovinos y caprinos (Craven y col 1999). Sobre resistencia a ivermectina de parásitos del bovino hay poca información: en Nueva Zelanda (West y col 1994, Vermunt y col 1995), en Inglaterra (Stafford y Coles 1999), en Argentina (Fiel y col 2000, Anziani y col 2001), y en Chile (Moenen-Locoz 1998, Sievers y Fuentealba 2003).

La Asociación Mundial para el Avance de la Parasitología Veterinaria (WAAVP) ha estandarizado las pruebas para detectar la resistencia antihelmíntica de nematodos (Coles y col 1992). Una de ellas es la prueba in vivo de reducción de la oviposición en la materia fecal FECRT (fecal egg count reduction test), que determina la eficacia antihelmíntica comparando la eliminación de huevos antes y después de un tratamiento. La otra prueba, muy laboriosa y de resultados inseguros, se realiza in vitro y consiste en determinar la inhibición del desarrollo (parálisis) de las larvas LDA (larval development assay) al ser sometidas a diferentes concentraciones de un producto determinado (Coles 1990, Hubert y Kerboeuf 1992, Ihler 1995).

El objetivo del presente trabajo es determinar resistencia antihelmíntica de los nematodos del bovino frente a la ivermectina, mediante la prueba FECRT en dos predios del sur de Chile. 


\section{MATERIAL Y METODOS}

El trabajo se realizó entre junio y agosto del año 2002 en dos predios de la X Región de Chile: Predio 1, cercano a Purranque $\left(40^{\circ} 53^{\prime} \mathrm{S}, 73^{\circ} 09^{\prime} \mathrm{O}\right)$, en el que se ha aplicado esporádicamente ivermectina al ganado vacuno, y Predio 2, cercano a San Pablo $\left(40^{\circ} 23^{\prime}\right.$ S, $73^{\circ} 02^{\prime}$ O), con aplicación regular, mínimo cada cuatro meses, de ivermectina a los bovinos menores de 1,5 años durante las últimas dos décadas. Al azar se eligieron en cada predio, respectivamente, 14 y 22 bovinos de 7 a 8 meses de edad para realizar la prueba de reducción de la oviposición (FECRT).

Rectalmente se obtuvieron muestras fecales de cada animal una semana antes (a.t.) y tres semanas postratamiento (p.t.) con $0,2 \mathrm{mg} / \mathrm{kg}$ de ivermectina ${ }^{*}$. A cada muestra se le realizó recuento de huevos (hpg) (Schmidt 1971) y un cultivo de larvas (Roberts y O’Sullivan 1950). El porcentaje de reducción de la oviposición se calculó mediante la fórmula (Young y col 1999):

$\%$ de reducción $=$ (Media hpg a.t. - Media hpg p.t.) $\times 100$ de la oviposición $=$ Media de hpg a.t.

\section{RESULTADOS Y DISCUSION}

Para la prueba (FECRT), una reducción de la oviposición después de un tratamiento inferior al 90\%, es indicativa de resistencia antihelmíntica de los parásitos involucrados (Coles y col 1992). Según este criterio, en el Predio 1 no hubo resistencia antihelmíntica al encontrarse un $90,3 \%$ de reducción de la oviposición, pero se puede deducir que debe estar en pleno proceso de selección de las cepas de nematodos resistentes, sien-

Cuadro 1. Promedio de huevos de nematodos diferenciados por gramo de heces antes y después del tratamiento con ivermectina (hpg a.t. y hpg p.t.) y porcentajes de reducción de la oviposición (\% red.) en bovinos de dos predios de la X Región, Chile.

Mean of differentiated nematode fecal egg count before and after a treatment with ivermectin (hpg a.t. and hpg p.t.), and percentage of reduction of the oviposition (\% red.) in cattle of two farms in the $10^{\text {th }}$ Region, Chile.

\begin{tabular}{lrrrrrr}
\hline Predios & \multicolumn{3}{c}{1} & & \multicolumn{2}{c}{2} \\
\cline { 2 - 7 } Género & hpg a.t. & hpg p.t. & \% red. & hpg a.t. & hpg p.t. & \% red. \\
\hline Ostertagia & 272,3 & 12,9 & 95,3 & 72,3 & 3,2 & 95,6 \\
Cooperia & 109,8 & 19,0 & 82,7 & 40,0 & 14,4 & 64,0 \\
Trichostrongylus & 55,3 & 10,7 & 68,0 & 12,7 & 2,9 & 77,1 \\
Nematodirus & 0,0 & 0,0 & 0,0 & 34,1 & 21,6 & 36,7 \\
Indiferenciadas & 1,8 & 0,2 & 88,9 & 0,0 & 0,0 & 0,0 \\
\hline Total & 439,2 & 42,8 & 90,3 & 159,1 & 42,1 & 73,5 \\
\hline
\end{tabular}

do Trichostrongylus y Cooperia los géneros más resistentes. En el Predio 2 hubo una clara resistencia antihelmíntica con un 73,6\% de reducción de la oviposición, con Nematodirus como el género más resistente seguido por Cooperia y Trichostrongylus (cuadro 1). Ello concuerda con lo observado en Chile (Moenen-Locoz 1998, Sievers y Fuentealba 2003), en Argentina (Fiel y col 2000, Anziani y col 2001), y en Nueva Zelanda (Vermunt y col 1995). Los resultados difieren con un trabajo realizado en Inglaterra (Armour y col 1980), pero ello puede deberse a que en ese entonces todavía no se había desarrollado resistencia antihelmíntica al producto.

Resistencia antihelmíntica se ha descrito en los nematodos de ovinos, caprinos y equinos, existiendo al respecto abundante literatura (FAO, 2003). Una de las razones que en esas especies se desarrolle más rápido la resistencia antihelmíntica puede deberse a la longevidad de sus parásitos, que supera los 150 días. De esta manera, al aplicar un producto sobre la población parasitaria, sobreviven algunos individuos y son sólo ellos los que producen la contaminación de las áreas de pastoreo durante un tiempo largo. En cambio, en la especie bovina los nematodos sólo viven 11 a 21 días como parásitos dentro del animal (Anderson, 1992) y, consecuentemente, los parásitos que sobreviven a un tratamiento logran contaminar mucho menos las pasturas, reduciéndose de esa forma la posibilidad de infecciones exitosas con las cepas de nematodos resistentes y el reemplazo de la población.

En ambos predios cerca de la mitad de los animales presentó una reducción de la oviposición superior al $90 \%$, lo cual indica que existen animales portadores de parásitos sensibles a la ivermectina. En el Predio 1 se detectó una resistencia moderada al producto porque no hubo bovinos con porcentajes de reducción de la oviposición inferiores al 40\%; esto se explica porque en el predio sólo esporádicamente se aplicó ivermectina a los bovinos. Sin embargo, en el Predio 2 hubo un $40,9 \%$ de los animales con porcentajes de reducción de la oviposición inferiores al 80\%, de los cuales casi la mitad tenía una reducción de la oviposición inferior al $40 \%$ (cuadro 2). La alta resistencia antihelmíntica frente a la ivermectina detectada en el predio 2 se concentró principalmente en el género Nematodirus seguido por Cooperia y Trichostrongylus; esto concuerda con observaciones previas hechas en Chile (Moenen-Locoz 1998, Sievers y Fuentealba 2003). En la primera prueba de eficacia de la ivermectina realizada en bovinos en Chile (Robles 1983), se detectó que Nematodirus seguía oviponiendo después del tratamiento, y es probable que esa resistencia innata de dicho género se haya magnificado en los últimos decenios por el extensivo y repetitivo uso de la ivermectina en el ganado vacuno del sur de Chile.

\footnotetext{
* IVOMEC (R), Merial.
} 
Cuadro 2. Distribución de los bovinos según los porcentajes de reducción de la oviposición de nematodos parásitos postratamiento con ivermectina de dos predios de la X Región, Chile.

Bovine distribution according to percentage of nematode parasite egg count reduction after treatment with ivermectin in two farms in the $10^{\text {th }}$ Region, Chile.

\begin{tabular}{lrrrr}
\hline \multirow{2}{*}{$\begin{array}{l}\text { \% Reducción } \\
\text { oviposición }\end{array}$} & \multicolumn{2}{c}{ Predio 1 } & \multicolumn{2}{c}{ Predio 2 } \\
\cline { 2 - 5 }$>90 \%$ & $\mathrm{n}$ & $\%$ & $\mathrm{n}$ & $\%$ \\
\hline $90-80 \%$ & 7 & 50,0 & 10 & 45,5 \\
$80-40 \%$ & 5 & 35,7 & 3 & 13,6 \\
$<40 \%$ & 2 & 14,3 & 5 & 22,7 \\
\hline Totales & 0 & 0,0 & 4 & 18,2 \\
\hline
\end{tabular}

El presente trabajo permite concluir que existe resistencia antihelmíntica de los géneros Nematodirus, Cooperia y Trichostrongylus frente a la ivermectina en un predio y que en el otro se está iniciando el proceso de selección de cepas resistentes.

\section{RESUMEN}

Resistencia antihelmíntica de nematodos frente a la ivermectina en bovinos se determinó en dos predios de la X Región del sur de Chile: uno cerca de Purranque (Predio 1) y otro cerca de San Pablo (Predio 2). Respectivamente, 14 y 22 bovinos de 7 a 8 meses de edad fueron tratados con $0,2 \mathrm{mg} / \mathrm{kg}$ de ivermectina. De cada animal se obtuvo materia fecal 7 días antes y 21 días después del tratamiento para realizar la prueba de reducción de la oviposición (FECRT). Se encontró un 90,3\% de reducción de la oviposición en el Predio 1, y los géneros más resistentes fueron Trichostrongylus y Cooperia. En el Predio 2 se encontró un 73,5\% de reducción de la oviposición con el género Nematodirus como el más resistente seguido por Cooperia y Trichostrongylus. Se concluyó que existe resistencia antihelmíntica frente a la ivermectina en los nematodos del bovino en el Predio 2 y que en el Predio 1 está en proceso de formación.

\section{REFERENCIAS}

Anderson, RC. 1992. Nematodes parasites in vertebrates. Their development and Transmission. CAB International, Wallingford, U.K.

Anziani O, G Zimmermann, A Guglielmone, R Vásquez, V Suárez. 2001. Avermectin resistance in Cooperia pectinata in cattle in Argentina. Vet Rec 149, 58-59.

Armour J, K Bairden, JM Preston. 1980. Anthelmintic efficiency of ivermectin against naturally acquired bovine gastrointestinal nematodes. Vet Rec 107, 226-227.

Coles GC. 1990. Recent advances in laboratory models for evaluation of helminth chemotherapy. Br Vet J 146, 113-119.
Coles GC, C Bauer, FHM Borgsteede, TR Klei, MA Taylor, PJ Waller. 1992. World Association for the Advancement of Veterinary Parasitology (WAAVP) methods for detection of anthelmintic resistance in nematodes of veterinary importance. Vet Parasitol $44,35-44$

Craven J, H Bjorn, EH Barnes, SA Henriksen, P Nansen. 1999. A comparison of in vitro tests and a faecal egg count reduction test in detecting anthelmintic resistance in horse strongyles. Vet Parasitol 85, 49-59.

FAO (2003). Resistencia a los antiparasitarios. Estado actual con énfasis en América Latina. Boletín 157. Roma.

Fiel C, C Saumel, P Estefan, E Rodríguez, G Salaberry. 2000. Resistencia de los nematodos Trichostrongylideos -Cooperia y Trichostrongylus - a tratamientos con avermectinas en bovinos de la Pampa Húmeda, Argentina. Rev Med Vet 81, 310-315.

Hubert J, D Kerboeuf. 1992. A microlarval development assay for the detection of anthelmintic resistance in sheep nematodes. Vet Rec $130,442-446$.

Ihler CF. 1995. Use of two in vitro methods in the detection of benzimidazole resistance in equine small strongyles (Cyathostoma spp.). Vet Parasitol 65, 117-125.

Jackson F. 1993. Anthelmintic resistance - the state of play. Br Vet J 149, 123-138.

Moenen-Locoz AS. 1998. Estudio comparativo de la efectividad de cinco productos comerciales que contienen Ivermectina frente a parásitos gastrointestinales del bovino. Tesis, M.V., Universidad Austral de Chile, Facultad de Ciencias Veterinarias, Valdivia, Chile.

Robles S. 1983. Efecto del fármaco Ivermectina (Ivomec) sobre la eliminación de huevos de parásitos gastrointestinales en las fecas de terneros en sus primeros meses de vida. Tesis, M.V., Universidad Austral de Chile, Facultad de Ciencias Veterinarias, Valdivia, Chile.

Roberts F, PJ O'Sullivan. 1950. Methods for eggs count and larval cultives for strongylus infesting the gastrointestinal tract for cattle. Austr J Agr Res 1, 99-102.

Romero J, Boero C, Vásquez R, Aristizábal MT, Baldo A. 1998. Estudio de la resistencia a antihelmínticos en majadas de la mesopotamia argentina. Rev Med Vet 70, 342-346.

Sangster NC. 1999. Pharmacology of anthelmintic resistance in cyathostomes: will it occur whith the avermectin/milbemycins? Vet Parasitol 85, 189-204.

Schmidt U. 1971. Parasitologische Kotuntersuchung durch ein neues Verdünnungsver-fahren. Tierärztl Umsch 26, 229-230.

Sievers G, Fuentealba C. 2003. Comparación de la efectividad antihelmíntica de seis productos comerciales que contienen lactonas macrocíclicas frente a nematodos gastrointestinales del bovino. Arch Med Vet 35, 81-88.

Stafford K, Coles GC. 1999. Nematode control practices and anthelmintic resistance in dairy calves in the south west of England. Vet Rec 144, 659-661.

Vermunt J, D West, W Pomroy. 1995. Multiple resistance to ivermectin and oxfendazole in Cooperia species of cattle in New Zealand. Vet Rec 143, 443-446.

West DJ, J Vermunt, W Pomroy, H Bentall. 1994. Inefficacy of ivermectin againts Cooperia spp infection in cattle. $N Z$ Vet $J 42$, 192-193.

Young K, V Garza, K Snowden, RJ Dobson, D Powel, TM Craig. 1999. Parasite diversity and anthelmintic resistance in two herds of horses. Vet Parasitol 85, 205-214. 